\title{
NEW INEQUALITIES FOR HERMITE-HADAMARD AND SIMPSON TYPE WITH APPLICATIONS
}

\author{
M. E. ÖZDEMİR AND ÇETİN YILDIZ
}

\begin{abstract}
In this paper, we obtain new bounds for the inequalities of Simpson and HermiteHadamard type for functions whose second derivatives absolute values are $P$-convex. Some applications for special means of real numbers are also given.
\end{abstract}

\section{Introduction}

Let $f: I \subset \mathbb{R} \rightarrow \mathbb{R}$ be a convex function defined on the interval $I$ of real numbers and $a, b \in I$, with $a<b$. The following inequality, known as the Hermite-Hadamard inequality for convex functions, holds:

$$
f\left(\frac{a+b}{2}\right) \leq \frac{1}{b-a} \int_{a}^{b} f(x) d x \leq \frac{f(a)+f(b)}{2} .
$$

Since the inequalities in (1.1) have been also known as Hadamard's inequalities. In this work, we shall call them the Hermite-Hadamard inequalities or $\mathrm{H}-\mathrm{H}$ inequalities, for simplicity.

In recent years many authors have established several inequalities connected to $\mathrm{H}-\mathrm{H}$ inequality. For recent results, refinements, counterparts, generalizations and new $\mathrm{H}-\mathrm{H}$ and Simpson type inequalities see the papers [2], [4], [5], [8], [9], [11], [12] and [13].

The following inequality is well known in the literature as Simpson's inequality.

Let $f:[a, b] \rightarrow \mathbb{R}$ be a four times continuously differentiable mapping on $(a, b)$ and $\left\|f^{(4)}\right\|_{\infty}=$ sup $\left|f^{(4)}(x)\right|<\infty$. Then, the following inequality holds: $x \in(a, b)$

$$
\left|\frac{1}{3}\left[\frac{f(a)+f(b)}{2}+2 f\left(\frac{a+b}{2}\right)\right]-\frac{1}{b-a} \int_{a}^{b} f(x) d x\right| \leq \frac{1}{2880}\left\|f^{(4)}\right\|_{\infty}(b-a)^{2} .
$$

In [7], S.S. Dragomir et.al., defined following new class of functions.

Corresponding author: Çetin Yildiz.

2010 Mathematics Subject Classification. 26A51, 26D10, $26 \mathrm{D} 15$.

Key words and phrases. $P$-convex functions, Hermite-Hadamard inequality, simpson inequality, power mean inequality. 
Definition 1. A function $f: I \subseteq \mathbb{R} \rightarrow \mathbb{R}$ is $P$ - convex function or that $\mathrm{f}$ belongs to the class of $P(I)$, if it is nonnegative and for all $x, y \in I$ and $\lambda \in[0,1]$, satisfies the following inequality;

$$
f(\lambda x+(1-\lambda) y) \leq f(x)+f(y) .
$$

$P(I)$ contain all nonnegative monotone convex and quasi convex functions.

In [1], Akdemir and Özdemir defined co-ordinaded $P$-convex functions and proved some inequalities and in [7], Dragomir et al., proved following inequalities of Hadamard's type for $P-$ convex functions.

Theorem 1. Let $f \in P(I), a, b \in I$, with $a<b$ and $f \in L_{1}[a, b]$. Then the following inequality holds.

$$
f\left(\frac{a+b}{2}\right) \leq \frac{2}{b-a} \int_{a}^{b} f(x) d x \leq 2[f(a)+f(b)] .
$$

In [6], Dragomir and Pearce have studied this type of inequalities for twice differential function with bounded second derivative and have obtained the following:

Theorem 2. Assume that $f: I \rightarrow R$ is continuous on $I$, twice differentiable on $I^{\circ}$ and there exist $k, K$ such that $k \leq f^{\prime \prime} \leq K$ on $I$. Then

$$
\frac{k}{3}\left(\frac{b-a}{2}\right)^{2} \leq \frac{f(a)+f(b)}{2}-\frac{1}{b-a} \int_{a}^{b} f(x) d x \leq \frac{K}{3}\left(\frac{b-a}{2}\right)^{2} .
$$

In [3], Cerone and Dragomir proved the following theorem:

Theorem 3. Let $f:[a, b] \rightarrow R$ be a twice differentiable mapping and suppose that $\gamma \leq f^{\prime \prime} \leq \Gamma$ for all $t \in(a, b)$. Then we have

$$
\frac{\gamma(b-a)^{2}}{24} \leq \frac{1}{b-a} \int_{a}^{b} f(x) d x-f\left(\frac{a+b}{2}\right) \leq \frac{\Gamma(b-a)^{2}}{24} .
$$

In [10], Sarıkaya et al. established following Lemma for twice differentiable mappings:

Lemma 1. Let $I \subset \mathbb{R}$ be an open interval, with $a<b$. If $f: I \rightarrow \mathbb{R}$ is a twice differentiable mapping such that $f^{\prime \prime}$ is integrable and $0 \leq \lambda \leq 1$. Then the following identity holds:

$$
(\lambda-1) f\left(\frac{a+b}{2}\right)-\lambda \frac{f(a)+f(b)}{2}+\frac{1}{b-a} \int_{a}^{b} f(x) d x=(b-a)^{2} \int_{0}^{1} k(t) f^{\prime \prime}(t a+(1-t) b) d t
$$

where

$$
k(t)= \begin{cases}\frac{1}{2} t(t-\lambda), & 0 \leq t \leq \frac{1}{2} \\ \frac{1}{2}(1-t)(1-\lambda-t), & \frac{1}{2} \leq t \leq 1 .\end{cases}
$$


The main purpose of this paper is to point out new estimations of the inequalities (1.1) and (1.2) and to apply them in special means of the real numbers.

\section{Main Results}

Using Lemma 1, we can obtain the following general integral inequalities for $P$-convex functions.

Theorem 4. Let $f: I \subset \mathbb{R} \rightarrow \mathbb{R}$ be a differentiable mapping on $I^{o}\left(I^{o}\right.$ is the interior of $I$ ) and $a, b \in I$ with $a<b$. If $\left|f^{\prime \prime}\right|$ is $P$-convex function, $0 \leq \lambda \leq 1$, then the following inequality holds:

$$
\begin{aligned}
& \left|(\lambda-1) f\left(\frac{a+b}{2}\right)-\lambda \frac{f(a)+f(b)}{2}+\frac{1}{b-a} \int_{a}^{b} f(x) d x\right| \\
& \quad \leq \begin{cases}\frac{(b-a)^{2}}{24}\left(8 \lambda^{3}-3 \lambda+1\right)\left\{\left|f^{\prime \prime}(a)\right|+\left|f^{\prime \prime}(b)\right|\right\}, & \text { for } 0 \leq \lambda \leq \frac{1}{2}\end{cases} \\
& \frac{(b-a)^{2}}{24}(3 \lambda-1)\left\{\left|f^{\prime \prime}(a)\right|+\left|f^{\prime \prime}(b)\right|\right\}, \quad \text { for } \frac{1}{2} \leq \lambda \leq 1 .
\end{aligned}
$$

Proof. From Lemma 1, we have

$$
\begin{aligned}
\left|(\lambda-1) f\left(\frac{a+b}{2}\right)-\lambda \frac{f(a)+f(b)}{2}+\frac{1}{b-a} \int_{a}^{b} f(x) d x\right| \\
\leq \frac{(b-a)^{2}}{2}\left[\int_{0}^{\frac{1}{2}}|t(t-\lambda)|\left|f^{\prime \prime}(t a+(1-t) b)\right| d t\right. \\
\left.\quad+\int_{\frac{1}{2}}^{1}|(1-t)(1-\lambda-t)|\left|f^{\prime \prime}(t a+(1-t) b)\right| d t\right] .
\end{aligned}
$$

We assume that $0 \leq \lambda \leq \frac{1}{2}$, then using the $P$-convexity of $\left|f^{\prime \prime}\right|$, we have

$$
\begin{aligned}
& \int_{0}^{\frac{1}{2}}|t(t-\lambda)|\left|f^{\prime \prime}(t a+(1-t) b)\right| d t \\
& \quad=\int_{0}^{\lambda} t(\lambda-t)\left|f^{\prime \prime}(t a+(1-t) b)\right| d t+\int_{\lambda}^{\frac{1}{2}} t(t-\lambda)\left|f^{\prime \prime}(t a+(1-t) b)\right| d t \\
& \quad \leq\left\{\left|f^{\prime \prime}(a)\right|+\left|f^{\prime \prime}(b)\right|\right\}\left[\int_{0}^{\lambda} t(\lambda-t) d t+\int_{\lambda}^{\frac{1}{2}} t(t-\lambda) d t\right] \\
& \quad=\left\{\left|f^{\prime \prime}(a)\right|+\left|f^{\prime \prime}(b)\right|\right\}\left(\frac{\lambda^{3}}{3}-\frac{\lambda}{8}+\frac{1}{24}\right) .
\end{aligned}
$$

Similarly, we write

$$
\begin{aligned}
\int_{\frac{1}{2}}^{1} & |(1-t)(1-\lambda-t)|\left|f^{\prime \prime}(t a+(1-t) b)\right| d t \\
\quad & =\int_{\frac{1}{2}}^{1-\lambda}(1-t)(1-\lambda-t)\left|f^{\prime \prime}(t a+(1-t) b)\right| d t+\int_{1-\lambda}^{1}(1-t)(t+\lambda-1)\left|f^{\prime \prime}(t a+(1-t) b)\right| d t
\end{aligned}
$$




$$
\begin{aligned}
& \leq\left\{\left|f^{\prime \prime}(a)\right|+\left|f^{\prime \prime}(b)\right|\right\}\left[\int_{\frac{1}{2}}^{1-\lambda}(1-t)(1-\lambda-t) d t+\int_{1-\lambda}^{1}(1-t)(t+\lambda-1) d t\right] \\
& =\left\{\left|f^{\prime \prime}(a)\right|+\left|f^{\prime \prime}(b)\right|\right\}\left(\frac{2(1-\lambda)^{3}}{3}+\lambda(1-\lambda)^{2}+\frac{7 \lambda}{8}-\frac{5}{8}\right) .
\end{aligned}
$$

Using (2.3) and (2.4) in (2.2), we see that first inequality of (2.1) holds.

On the other hand, let $\frac{1}{2} \leq \lambda \leq 1$, then, from $P$-convexity of $\left|f^{\prime \prime}\right|$ we have

$$
\begin{aligned}
& \int_{0}^{\frac{1}{2}}|t(t-\lambda)|\left|f^{\prime \prime}(t a+(1-t) b)\right| d t+\int_{\frac{1}{2}}^{1}|(1-t)(1-\lambda-t)|\left|f^{\prime \prime}(t a+(1-t) b)\right| d t \\
& \quad \leq\left\{\left|f^{\prime \prime}(a)\right|+\left|f^{\prime \prime}(b)\right|\right\}\left[\int_{0}^{\frac{1}{2}} t(\lambda-t) d t+\int_{\frac{1}{2}}^{1}(1-t)(t+\lambda-1) d t\right] \\
& \quad=\left\{\left|f^{\prime \prime}(a)\right|+\left|f^{\prime \prime}(b)\right|\right\}\left(\frac{\lambda}{4}-\frac{1}{12}\right) .
\end{aligned}
$$

This is second inequality of (2.1). This completes the proof.

Theorem 5. Let $f: I \subset \mathbb{R} \rightarrow \mathbb{R}$ be a differentiable mapping on $I^{o}$ and $a, b \in I$ with $a<b$. If $\left|f^{\prime \prime}\right|^{q}$ is $P$-convex function, $0 \leq \lambda \leq 1$ and $q \geq 1$, then the following inequality holds:

$$
\begin{aligned}
& \left|(\lambda-1) f\left(\frac{a+b}{2}\right)-\lambda \frac{f(a)+f(b)}{2}+\frac{1}{b-a} \int_{a}^{b} f(x) d x\right| \\
& \quad \leq \begin{cases}\frac{(b-a)^{2}}{48}\left(8 \lambda^{3}-3 \lambda+1\right)\left(\left\{\left|f^{\prime \prime}(a)\right|^{q}+\left|f^{\prime \prime}(b)\right|^{q}\right\}\right)^{\frac{1}{q}}, & \text { for } 0 \leq \lambda \leq \frac{1}{2} \\
\frac{(b-a)^{2}}{48}(3 \lambda-1)\left(\left\{\left|f^{\prime \prime}(a)\right|^{q}+\left|f^{\prime \prime}(b)\right|^{q}\right\}\right)^{\frac{1}{q}}, & \text { for } \frac{1}{2} \leq \lambda \leq 1\end{cases}
\end{aligned}
$$

Proof. From Lemma 1 and using well known power mean inequality, we get

$$
\begin{aligned}
&\left|(\lambda-1) f\left(\frac{a+b}{2}\right)-\lambda \frac{f(a)+f(b)}{2}+\frac{1}{b-a} \int_{a}^{b} f(x) d x\right| \\
& \leq \frac{(b-a)^{2}}{2}\left[\int_{0}^{\frac{1}{2}}|t(t-\lambda)|\left|f^{\prime \prime}(t a+(1-t) b)\right| d t\right. \\
&\left.+\int_{\frac{1}{2}}^{1}|(1-t)(1-\lambda-t)|\left|f^{\prime \prime}(t a+(1-t) b)\right| d t\right] \\
& \leq \frac{(b-a)^{2}}{2}\left(\int_{0}^{\frac{1}{2}}|t(t-\lambda)| d t\right)^{1-\frac{1}{q}}\left(\int_{0}^{\frac{1}{2}}|t(t-\lambda)|\left[\left|f^{\prime \prime}(t a+(1-t) b)\right|\right]^{q} d t\right)^{\frac{1}{q}} \\
&+\left(\int_{\frac{1}{2}}^{1}|(1-t)(1-\lambda-t)| d t\right)^{1-\frac{1}{q}}\left(\int_{\frac{1}{2}}^{1}|(1-t)(1-\lambda-t)|\left[\left|f^{\prime \prime}(t a+(1-t) b)\right|\right]^{q} d t\right)^{\frac{1}{q}} .
\end{aligned}
$$

Let $0 \leq \lambda \leq \frac{1}{2}$. Since $\left|f^{\prime \prime}\right|$ is $P$-convex on $[a, b]$, we write

$$
\int_{0}^{\frac{1}{2}}|t(t-\lambda)|\left[\left|f^{\prime \prime}(t a+(1-t) b)\right|\right]^{q} d t
$$




$$
\begin{aligned}
& =\int_{0}^{\lambda} t(\lambda-t)\left[\left|f^{\prime \prime}(t a+(1-t) b)\right|\right]^{q} d t+\int_{\lambda}^{\frac{1}{2}} t(t-\lambda)\left[\left|f^{\prime \prime}(t a+(1-t) b)\right|\right]^{q} d t \\
\leq & \left\{\left|f^{\prime \prime}(a)\right|^{q}+\left|f^{\prime \prime}(b)\right|^{q}\right\}\left[\int_{0}^{\lambda} t(\lambda-t) d t+\int_{\lambda}^{\frac{1}{2}} t(t-\lambda) d t\right] \\
= & \left\{\left|f^{\prime \prime}(a)\right|^{q}+\left|f^{\prime \prime}(b)\right|^{q}\right\}\left(\frac{\lambda^{3}}{3}-\frac{\lambda}{8}+\frac{1}{24}\right), \\
\int_{\frac{1}{2}}^{1}|(1-t)(1-\lambda-t)|\left[\left|f^{\prime \prime}(t a+(1-t) b)\right|\right]^{q} d t & \int_{\frac{1}{2}}^{1-\lambda}(1-t)(1-\lambda-t)\left[\left|f^{\prime \prime}(t a+(1-t) b)\right|\right]^{q} d t \\
& +\int_{1-\lambda}^{1}(1-t)(t+\lambda-1)\left[\left|f^{\prime \prime}(t a+(1-t) b)\right|\right]^{q} d t \\
\leq & \left\{\left|f^{\prime \prime}(a)\right|^{q}+\left|f^{\prime \prime}(b)\right|^{q}\right\}\left[\int_{\frac{1}{2}}^{1-\lambda}(1-t)(1-\lambda-t) d t+\int_{1-\lambda}^{1}(1-t)(t+\lambda-1) d t\right] \\
= & \left\{\left|f^{\prime \prime}(a)\right|^{q}+\left|f^{\prime \prime}(b)\right|^{q}\right\}\left(\frac{2(1-\lambda)^{3}}{3}+\lambda(1-\lambda)^{2}+\frac{7 \lambda}{8}-\frac{5}{8}\right), \\
\int_{0}^{\frac{1}{2}}|t(t-\lambda)| d t=\int_{0}^{\lambda} t(\lambda-t) d t+\int_{\lambda}^{\frac{1}{2}} t(t-\lambda) d t=\frac{\lambda^{3}}{3}+\frac{1-3 \lambda}{24} &
\end{aligned}
$$

and

$$
\begin{aligned}
\int_{\frac{1}{2}}^{1}|(1-t)(1-\lambda-t)| d t & =\int_{\frac{1}{2}}^{1-\lambda}(1-t)(1-\lambda-t) d t+\int_{1-\lambda}^{1}(1-t)(t+\lambda-1) d t \\
& =\frac{\lambda^{3}}{3}+\frac{1-3 \lambda}{24} .
\end{aligned}
$$

Thus, using (2.7)-(2.10) in (2.6), we obtain the first inequality of (2.5).

Now, let $\frac{1}{2} \leq \lambda \leq 1$, then, using the $P$-convexity of $\left|f^{\prime \prime}\right|^{q}$, we have

$$
\begin{aligned}
\int_{0}^{\frac{1}{2}}|t(t-\lambda)|\left[\left|f^{\prime \prime}(t a+(1-t) b)\right|\right]^{q} d t & =\int_{0}^{\frac{1}{2}} t(\lambda-t)\left[\left|f^{\prime \prime}(t a+(1-t) b)\right|\right]^{q} d t \\
& \leq \int_{0}^{\frac{1}{2}} t(\lambda-t)\left\{\left|f^{\prime \prime}(a)\right|^{q}+\left|f^{\prime \prime}(b)\right|^{q}\right\} d t \\
& =\left\{\left|f^{\prime \prime}(a)\right|^{q}+\left|f^{\prime \prime}(b)\right|^{q}\right\}\left(\frac{\lambda}{8}-\frac{1}{24}\right),
\end{aligned}
$$

similarly,

$$
\begin{aligned}
\int_{\frac{1}{2}}^{1}|(1-t)(1-\lambda-t)|\left[\left|f^{\prime \prime}(t a+(1-t) b)\right|\right]^{q} d t & =\int_{\frac{1}{2}}^{1}(1-t)(t+\lambda-1)\left[\left|f^{\prime \prime}(t a+(1-t) b)\right|\right]^{q} d t \\
& \leq \int_{\frac{1}{2}}^{1}(1-t)(t+\lambda-1)\left\{\left|f^{\prime \prime}(a)\right|^{q}+\left|f^{\prime \prime}(b)\right|^{q}\right\} d t
\end{aligned}
$$




$$
=\left\{\left|f^{\prime \prime}(a)\right|^{q}+\left|f^{\prime \prime}(b)\right|^{q}\right\}\left(\frac{\lambda}{8}-\frac{1}{24}\right)
$$

We also have

$$
\int_{0}^{\frac{1}{2}}|t(t-\lambda)| d t=\int_{\frac{1}{2}}^{1}|(1-t)(1-\lambda-t)| d t=\frac{3 \lambda-1}{24} .
$$

Therefore, if we use the (2.11), (2.12) and (2.13) in (2.6), we obtain the second inequality of (2.5). This completes the proof.

Corollary 1. In Theorem 5 , if we choose $\lambda=0$, we obtain

$$
\left|\frac{1}{b-a} \int_{a}^{b} f(x) d x-f\left(\frac{a+b}{2}\right)\right| \leq \frac{(b-a)^{2}}{48}\left(\left\{\left|f^{\prime \prime}(a)\right|^{q}+\left|f^{\prime \prime}(b)\right|^{q}\right\}\right)^{\frac{1}{q}}
$$

which similar to the left hand side of H-H inequality.

Corollary 2. In Theorem 5 we choose $\lambda=1$, we obtain

$$
\left|\frac{f(a)+f(b)}{2}-\frac{1}{b-a} \int_{a}^{b} f(x) d x\right| \leq \frac{(b-a)^{2}}{24}\left(\left\{\left|f^{\prime \prime}(a)\right|^{q}+\left|f^{\prime \prime}(b)\right|^{q}\right\}\right)^{\frac{1}{q}}
$$

which similar to the right hand side of $\mathrm{H}$-H inequality.

Corollary 3. In Theorem 5 , if we choose $\lambda=\frac{1}{3}$, we obtain

$$
\left|\frac{1}{3}\left[\frac{f(a)+f(b)}{2}+2 f\left(\frac{a+b}{2}\right)\right]-\frac{1}{b-a} \int_{a}^{b} f(x) d x\right| \leq \frac{(b-a)^{2}}{162}\left(\left\{\left|f^{\prime \prime}(a)\right|^{q}+\left|f^{\prime \prime}(b)\right|^{q}\right\}\right)^{\frac{1}{q}}
$$

which similar to the Simpson inequality.

Furthermore if $f^{\prime \prime}$ is bounded on $I=[a, b]$ then we have the following corollary:

Corollary 4. In Corollary 1 , if $\left|f^{\prime \prime}\right| \leq M, M>0$, then we have

$$
\left|\frac{1}{b-a} \int_{a}^{b} f(x) d x-f\left(\frac{a+b}{2}\right)\right| \leq M \frac{(b-a)^{2}}{48} 2^{\frac{1}{q}} .
$$

Since $2^{\frac{1}{q}} \leq 2$ for $q \geq 1$, we obtain

$$
\left|\frac{1}{b-a} \int_{a}^{b} f(x) d x-f\left(\frac{a+b}{2}\right)\right| \leq M \frac{(b-a)^{2}}{24}
$$

which is (1.4) inequality.

Corollary 5. In Corollary 2, if $\left|f^{\prime \prime}\right| \leq M, M>0$, then we have

$$
\left|\frac{f(a)+f(b)}{2}-\frac{1}{b-a} \int_{a}^{b} f(x) d x\right| \leq M \frac{(b-a)^{2}}{24} 2^{\frac{1}{q}} .
$$

Since $2^{\frac{1}{q}} \leq 2$ for $q \geq 1$, we obtain

$$
\left|\frac{f(a)+f(b)}{2}-\frac{1}{b-a} \int_{a}^{b} f(x) d x\right| \leq M \frac{(b-a)^{2}}{12}
$$

which is (1.3) inequality. 
Corollary 6. In Corollary 3, if $\left|f^{\prime \prime}\right| \leq M, M>0$, then we have

$$
\left|\frac{1}{3}\left[\frac{f(a)+f(b)}{2}+2 f\left(\frac{a+b}{2}\right)\right]-\frac{1}{b-a} \int_{a}^{b} f(x) d x\right| \leq M \frac{(b-a)^{2}}{162} 2^{\frac{1}{q}} .
$$

Since $2^{\frac{1}{q}} \leq 2$ for $q \geq 1$, we obtain

$$
\left|\frac{1}{3}\left[\frac{f(a)+f(b)}{2}+2 f\left(\frac{a+b}{2}\right)\right]-\frac{1}{b-a} \int_{a}^{b} f(x) d x\right| \leq M \frac{(b-a)^{2}}{81} .
$$

\section{Applications to Special Means}

We now consider the means for arbitrary real numbers $\alpha, \beta(\alpha \neq \beta)$. We take

1. Arithmetic mean:

$$
A(\alpha, \beta)=\frac{\alpha+\beta}{2}, \alpha, \beta \in \mathbb{R}^{+}
$$

2. Logarithmic mean:

$$
L(\alpha, \beta)=\frac{\alpha-\beta}{\ln |\alpha|-\ln |\beta|}, \quad|\alpha| \neq|\beta|, \alpha, \beta \neq 0, \alpha, \beta \in \mathbb{R}^{+} .
$$

3. Generalized log-mean:

$$
L_{n}(\alpha, \beta)=\left[\frac{\beta^{n+1}-\alpha^{n+1}}{(n+1)(\beta-\alpha)}\right]^{\frac{1}{n}}, \quad n \in \mathbb{Z} \backslash\{-1,0\}, \alpha, \beta \in \mathbb{R}^{+} .
$$

Now using the results of Section 2, we give some applications for special means of real numbers.

Proposition 6. Let $a, b \in \mathbb{R}, 0<a<b$ and $n \in \mathbb{Z},|n(n-1)| \geq 3$, then, for all $q \geq 1$, the following inequality holds:

$$
\left|L_{n}^{n}(a, b)-A^{n}(a, b)\right| \leq|n(n-1)| \frac{(b-a)^{2}}{48}\left(\left\{a^{q(n-2)}+b^{q(n-2)}\right\}\right)^{\frac{1}{q}} .
$$

Proof. The proof is obvious from Corollary 4 applied to the $P$-convex mapping $f(x)=x^{n}$, $x \in[a, b], n \in \mathbb{Z}$.

Proposition 7. Let $a, b \in \mathbb{R}, 0<a<b$ and $n \in \mathbb{Z},|n(n-1)| \geq 3$, then, for all $q \geq 1$, the following inequality holds:

$$
\left|A\left(a^{n}, b^{n}\right)-L_{n}^{n}(a, b)\right| \leq|n(n-1)| \frac{(b-a)^{2}}{24}\left(\left\{a^{q(n-2)}+b^{q(n-2)}\right\}\right)^{\frac{1}{q}} .
$$


Proof. The proof is obvious from Corollary 6 applied to the $P$-convex mapping $f(x)=x^{n}$, $x \in[a, b], n \in \mathbb{Z}$.

Proposition 8. Let $a, b \in \mathbb{R}, 0<a<b$ and $n \in \mathbb{Z},|n(n-1)| \geq 3$, then, for all $q \geq 1$, the following inequality holds:

$$
\left|\frac{1}{3} A\left(a^{n}, b^{n}\right)+\frac{2}{3} A^{n}(a, b)-L_{n}^{n}(a, b)\right| \leq|n(n-1)| \frac{(b-a)^{2}}{162}\left(\left\{a^{q(n-2)}+b^{q(n-2)}\right\}\right)^{\frac{1}{q}} .
$$

Proof. The proof is obvious from Corollary 8 applied to the $P$-convex mapping $f(x)=x^{n}$, $x \in[a, b], n \in \mathbb{Z}$.

\section{References}

[1] A. O. Akdemir and M. E. Özdemir, Some Hadamard-Type inequalities for coordinated P-convex functions and Godunova-Levin functions, AIP Conference Proceedings, 1309(2010), 7-15.

[2] M. Alomari, M. Darus and S. S. Dragomir, New inequalities of Simpson's type for s-convex functions with applications, RGMIA Res. Rep. Coll., 12(4) (2009), Article 9.

[Online:http://www.staff.vu.edu.au/RGMIA/v12n4.asp]

[3] P. Cerone and S. S. Dragomir, Midpoint-type rules from an inequality point of view, Handbook of AnalyticComputational Methods in Applied Mathematics, Editor: G. Anastassiou, CRC Press, New York, 2000, 135200.

[4] S. S. Dragomir, Two mappings in connection to Hadamard's inequalities, J. Math. Anal. Appl., 167(1992), 4956.

[5] S. S. Dragomir, R. P. Agarwal and P. Cerone, On Simpson's inequality and applications, J. of Inequal. Appl., 5(2000), 533-579.

[6] S. S. Dragomir and C. E. M. Pearce, Selected Topics on Hermite-Hadamard Inequalities, (RGMIA Monographs http:// rgmia.vu.edu.au/ monographs/ hermite hadamard.html), Victoria University, 2000.

[7] S. S. Dragomir, J. Pecaric and L. E. Persson, Some inequalities of Hadamard Type, Soochow Journal of Mathematics, 21 (1995), 335-341.

[8] U. S. Kirmaci, Inequalities for differentiable mappings and applications to special means of real numbers to midpoint formula, Appl. Math. Comp., 147(2004), 137-146.

[9] B. Z. Liu, An inequality of Simpson type, Proc. R. Soc. A., 461 (2005), 2155-2158.

[10] M. Z. Sarıkaya and N. Aktan, On the generalization some intgeral inequalities and their applications, Math. and Comp. Mod., 54(2011), 2175-2182.

[11] E. Set, M. E. Özdemir and M.Z. Sarıaya, On new inequalities of Simpson's type for quasi-convex functions with applications, Tamkang. J. Math., 43(2012), 357-364.

[12] Ç. Yıldız, A. O. Akdemir, M. Avcı, New inequalities of Hermite-Hadamard type for functions whose derivatives absolute values are quasi convex, Erzincan University Graduate School of Natural and Applied Sciences Institute Journal, 3(2010), 263-272.

[13] G. S. Yong, D. Y. Hwang and K. L. Tseng, Some inequalities for differentiable convex and concave mappings, Comp. Math. Appl., 47(2004), 207-216.

AtatÜRk University, K. K. Education Faculty, Department of Mathematics, 25240, Campus, Erzurum, Turkey. E-mail: emos@atauni.edu.tr

AtatÜRk University, K. K. Education Faculty, Department of Mathematics, 25240, Campus, Erzurum, Turkey. E-mail: yildizcetiin@yahoo.com 